\title{
Current treatment status and medical costs for hemodialysis vascular access based on analysis of the Korean Health Insurance Database
}

Hyung Seok Lee ${ }^{1}$, Young-Su Ju², Young Rim Song ${ }^{1}$, Jwa Kyung Kim ${ }^{1}$, Sun Ryoung Choi ${ }^{1}$, Narae Joo ${ }^{1}$, Hyung Jik Kim ${ }^{1}$, Pyoungju Park ${ }^{1}$, and Sung Gyun Kim ${ }^{1}$

${ }^{1}$ Department of Internal Medicine, Kidney Research Institute, ${ }^{2}$ Department of Occupational and Environmental Medicine, Hallym University Sacred Heart Hospital, Anyang, Korea
Received: May 12, 2018
Revised : July 2, 2018
Accepted: July 24, 2018

\section{Correspondence to}

Sung Gyun Kim, M.D.

Department of Internal Medicine, Kidney Research Institute, Hallym University Sacred Heart Hospital, 22 Gwanpyeong-ro 17obeon-gil, Dongan-gu, Anyang 14068, Korea

Tel: +82-31-380-1942

Fax: +82-31-386-2269

E-mail: imnksk@hallym.or.kr
Background/Aims: The Republic of Korea is a country where the hemodialysis population is growing rapidly. It is believed that the numbers of treatments related to vascular access-related complications are also increasing. This study investigated the current status of treatment and medical expenses for vascular access in Korean patients on hemodialysis.

Methods: This was a descriptive observational study. We inspected the insurance claims of patients with chronic kidney disease who underwent hemodialysis between January 2008 and December 2016. We calculated descriptive statistics of the frequencies and medical expenses of procedures for vascular access.

Results: The national medical expenses for access-related treatment were 7.12 billion KRW (equivalent to 6.36 million USD) in 2008, and these expenses increased to 42.12 billion KRW (equivalent to 37.67 million USD) in 2016. The population of hemodialysis patients, the annual frequency of access-related procedures, and the total medical cost for access-related procedures increased by 1.6-, 2.6-, and 5.9-fold, respectively, over the past 9 years. The frequency and costs of access care increased as the number of patients on hemodialysis increased. The increase in vascular access-related costs has largely been driven by increased numbers of percutaneous angioplasty.

Conclusions: The increasing proportion of medical costs for percutaneous angioplasty represents a challenge in the management of end-stage renal disease in Korea. It is essential to identify the clinical and physiological aspects as well as anatomical abnormalities before planning angioplasty. A timely surgical correction could be a viable option to control the rapid growth of access-related medical expenses.

Keywords: Renal dialysis; Arteriovenous fistula; Endovascular procedures; Angioplasty; Administrative claims, healthcare

\section{INTRODUCTION}

The population of patients with end-stage renal disease (ESRD) who need hemodialysis (HD) treatment has in- creased over the past decade. Recently in the Republic of Korea, the proportion of patients with ESRD who received peritoneal dialysis decreased while that of ESRD patients who receive HD sharply increased [1]. These 
changes have resulted in an increased incidence of vascular access-related complications and increased medical costs for vascular access-related treatments. The Korean Society of Nephrology launched the End-Stage Renal Disease Patient Registry in 1985, but it has not yet provided a report on medical expenses and the trends related to managing the complications of vascular access.

South Korea has a National Health Insurance (NHI) system, which is a public single-payer system. The National Health Insurance Corporation (NHIC) is the only public insurance institution operated by the Ministry of Health and Welfare in this country. Healthcare providers are automatically eligible and obliged to treat patients with those services covered by the system. Every Korean resident is eligible regardless of nationality or profession. The coverage provided is comprehensive, including prevention, diagnosis, treatment, surgery, hospitalization, and rehabilitation. Insurance benefits for patients and payments to healthcare providers are supervised by the Health Insurance Review and Assessment Service (HIRA), which reviews insurance claims and assesses the healthcare services [2]. These characteristics of the NHI system make the claims data valid for studying medical service use and costs. We analyzed the health insurance claims data from the HIRA to investigate the current status of treatments for access-related complications and their associated medical expenses in patients on HD in the Republic of Korea.

\section{METHODS}

The Institutional Review Board of the Hallym University Sacred Heart Hospital approved this study (IRB number: HUSHHIRB 2017-Io79). We analyzed the records of patients with ESRD who underwent HD between January 2008 and December 2016 in the health insurance claims database. The diagnosis codes were standardized according to the Korean Classification of Disease, 6th version, which follows the International Classification of Disease, 1oth edition (ICD-10) [3]. We defined ESRD patients who underwent $\mathrm{HD}$ as those with a diagnosis of chronic kidney disease (CKD) (diagnosis codes: N18 or N19) and HD treatment (procedure codes: O7020 or O9991) or with the "rare/intractable diseases" code for HD (Voo1) (Fig. 1).

When patients in the NHI system receive healthcare, they pay a "co-payment" to the provider. Typical co-payment amounts are $20 \%$ for inpatient hospital care and $30 \%$ to $50 \%$ for outpatient care. The co-payment amount for patients with the rare/intractable diseases code for $\mathrm{HD}$ is $10 \%$ of inpatient and outpatient medical costs. Patients with ESRD who start HD are eligible for the rare/intractable diseases code if they undergo outpatient treatment on the day of HD or are hospitalized because of the ESRD or the HD. Thus, the rare/intractable diseases code is a useful tool to identify claims data related to examinations or treatments related to ESRD in patients on HD. We identified patients that had any procedure codes related to the following vascular access procedures: venography, fistulography, percutaneous

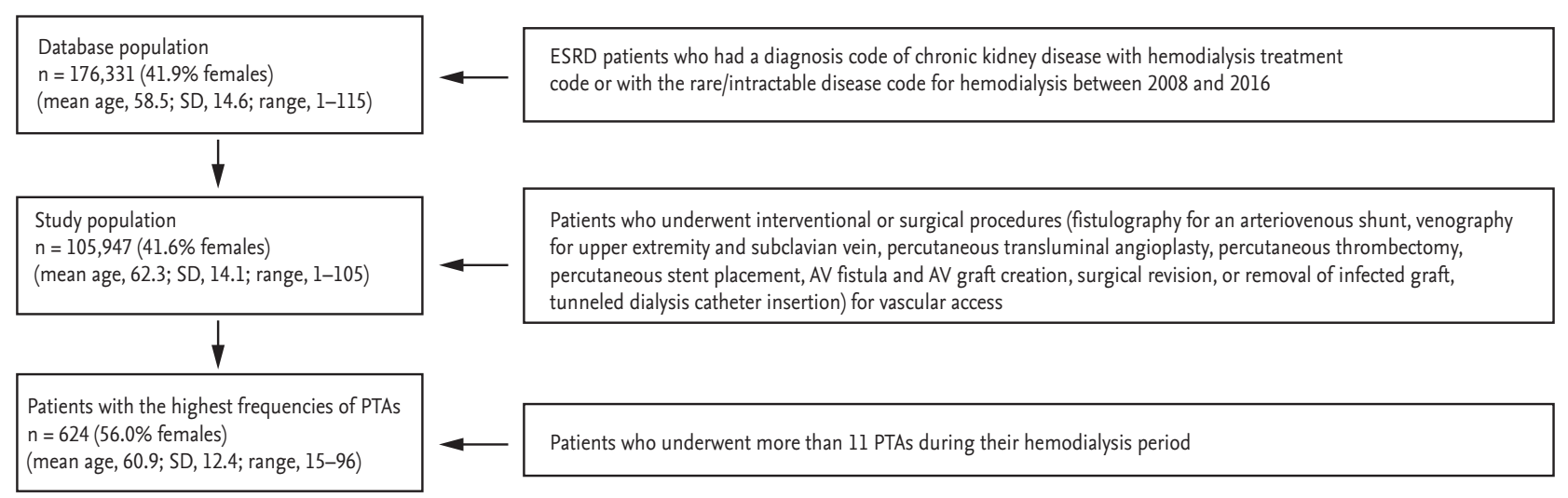

Figure 1. Diagram of the population selection. SD, standard deviation, ESRD, end-stage renal disease; AV, arteriovenous; PTA, percutaneous transluminal angioplasty. 
transluminal angioplasty (PTA), percutaneous thrombectomy, stent placement, surgical revision, arteriovenous (AV) fistula/graft placement, removal of infected graft, or tunneled dialysis catheter (TDC) placement. After excluding the treatment data not related to the vascular access of $\mathrm{HD}$, such as angioplasty for renal arteries and stent placement for iliofemoral arteries, we analyzed the medical cost of each access-related procedure. To investigate the number of treatments for the access-related complications, we eliminated duplicated procedural activities. For example, when a fistulography was performed during PTA, it was considered a case of PTA, and when a PTA was performed during percutaneous thrombectomy, it was considered a case of percutaneous thrombectomy. A total of $77 \%$ of fistulography cases were performed during PTA, and 10\% of PTA cases were performed during percutaneous thrombectomy in this study; however, they were not included in the analysis of the number of treatments. Therefore, the annual frequency of PTA cases analysis included cases where PTA was performed without thrombectomy and stent placement. We performed statistical analyses using SAS software version 9.4 (SAS Institute, Cary, NC, USA). We presented categorical variables as frequency and percentage and continuous variables as mean and standard deviation (SD).

\section{RESULTS}

We identified 176,331 (41.9\% female) patients with CKD who underwent HD between January 2006 and December 2016. The mean age was 58.5 years (SD, 14.6; median, 6o; Q1-Q3, 49 to 70; range, 1 to 115). The annual number of HD patients increased 1.57-fold from 49,403 in 2008 to 77,933 in 2016 . The total medical cost of access-related procedures increased 5.9-fold from 7.12 billion KRW (equivalent to 6.36 million USD) in 2008 to 42.12 billion KRW (equivalent to 37.67 million USD) in 2016. The medical cost of PTA increased 13-fold from 1.29 billion KRW (equivalent to 1.16 million USD) in 2008 to 17.02 billion KRW (equivalent to 15.22 million USD) in 2016 . The cost of PTA was the main driver of the increase in HD vascular access-related expenses. The total medical cost for access-related treatment rose more steeply in recent years, reaching 36.10 billion KRW (equivalent to 32.29 million USD) in 2015 and 42.12 billion KRW (equivalent to 37.67 million USD) in 2016 (Fig. 2, Supplementary Table 1). The total number of procedures for vascular access (i.e., venography, fistulography, PTA, percutaneous thrombectomy, stent placement, access creation, surgical revision, removal of infected graft, and TDC placement) increased by more than 2.6-fold over 9 years. As the number of patients on HD increased, the numbers of interventional and surgical procedures related to vascular access increased. The increase in the number of PTA procedures was especially remarkable, leading the growth in the numbers of procedures (Fig. 3, Supplementary Table 2). During the past 9 years, the annual frequency of PTA increased from $14.4 \%$ to $26.5 \%$ of the total frequency of treatment procedures, whereas the medical cost of PTA increased from $18.2 \%$ to $40.4 \%$ (Fig. 4).

Based on the annual number of patients on HD in the present study, we analyzed the annual frequency and medical costs of each procedure per patient on HD. This per-patient analysis showed growth in the number of PTAs per patient as well as the growth of the overall size of the population of patients on HD, which increased the annual number of PTAs. The annual frequency of PTA per patient has risen rapidly in recent years, and the frequency per patient per year increased from 0.1715 in 2013 to 0.1946 in 2014, 0.2055 in 2015 , and 0.2261 in 2016. However, the frequency per patient per year of surgical revision gradually decreased from 0.0871 in 2013 to 0.0864 in 2014, 0.0856 in 2015, and 0.0852 in 2016 (Fig. 5, Supplementary Tables 3 and 4).

Ten percent of the total PTAs (9,110 cases) were performed in patients with the highest frequencies of PTAs ( $n=624 ; 56 \%$ females; mean age, 60.5 years; range, 13 to 98 years). This patient group accounted for $1.7 \%$ of those who underwent PTA. The highest PTA frequency group underwent at least 11 PTAs during their dialysis period and had an average of 14.6 PTA treatments (range, 11 to 85; SD, 5.24; 95\% confidence interval, 14.18 to 15.01$)$. The average time from the first PTA to the eleventh PTA was 4.27 years. As the patients underwent repeated PTAs, the interval between procedures decreased and converged on 80 to 90 days. The most common time interval between PTAs was 90 days (Fig. 6 and Supplementary Figs. $1-8)$. 


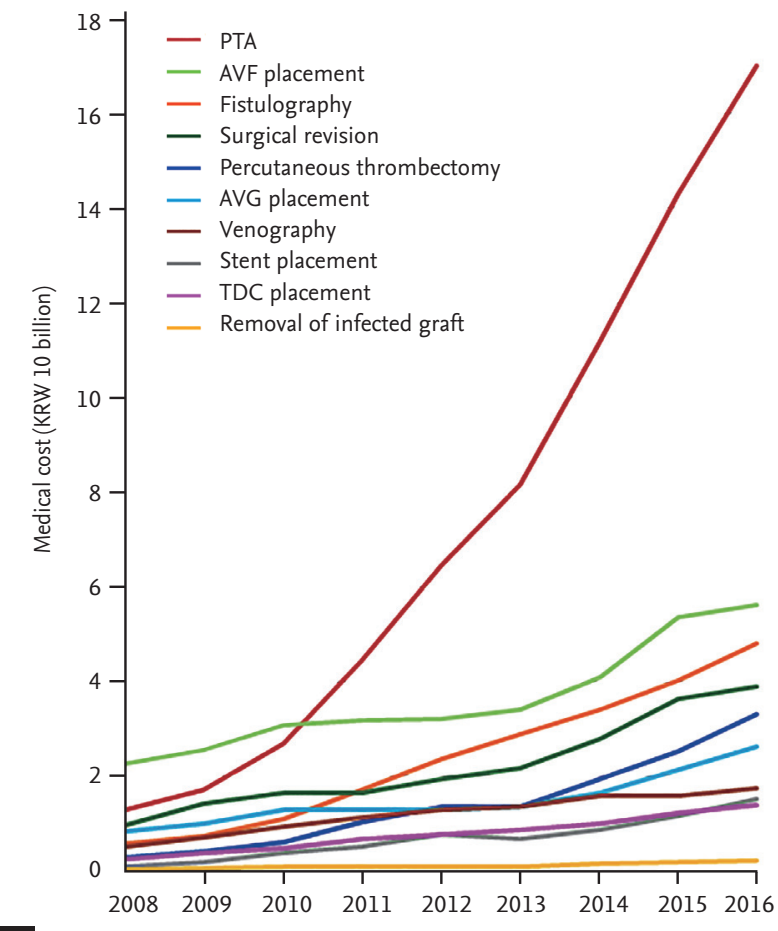

A $\begin{array}{lllllllll}2008 & 2009 & 2010 & 2011 & 2012 & 2013 & 2014 & 2015 & 2016\end{array}$

Year

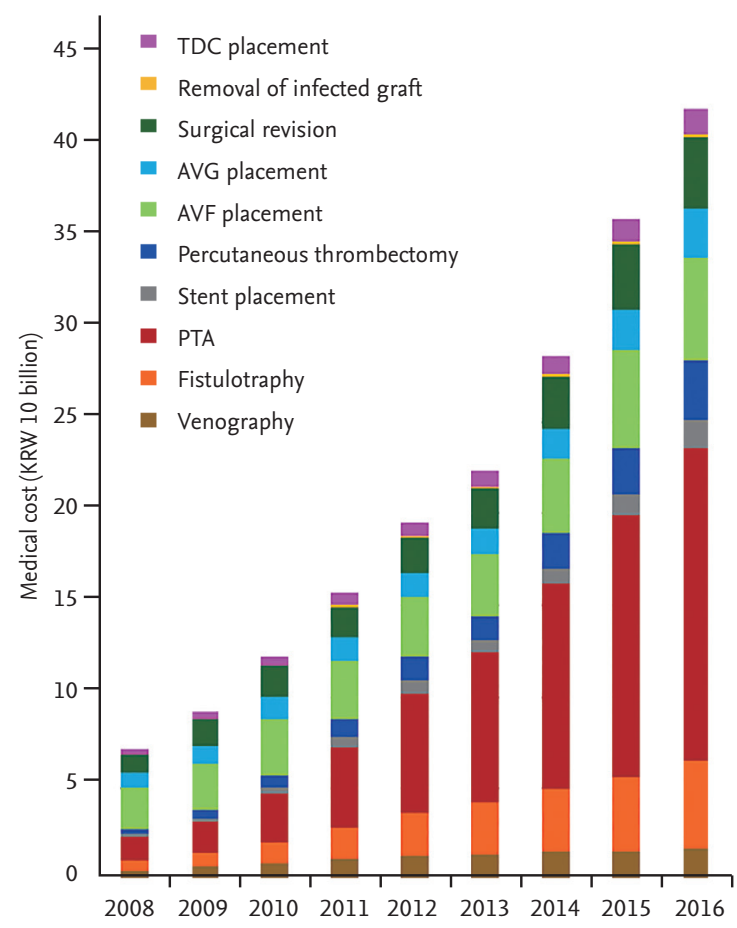

B

Year

Figure 2. Annual medical costs of vascular access-related treatments (2008 to 2016). (A) Trend in annual medical costs of access-related procedures. The annual medical cost of each procedure was the sum of the procedure costs that included the unit cost and the adjusted rate, such as the reoperation discount or holiday surcharge. (B) Trends in the proportion of medical costs of access-related treatments. The expenses for vascular access care increased along with the increase in the number of patients on hemodialysis. The greatest increase was in the costs for percutaneous angioplasty. PTA, percutaneous transluminal angioplasty; AVF, arteriovenous fistula; AVG, arteriovenous graft; TDC, tunneled dialysis catheter.

\section{DISCUSSION}

Both the overall number and the proportion of patients on HD has increased in the Republic of Korea. Patients on HD comprised over $90 \%$ of the patients on dialysis in this country in 2016. The incidence of vascular access-related complications and the number of procedures to treat these complications are thought to be increasing; however, there has been little or no investigation into the current treatment status and medical expenses of vascular access-related complications in the Republic of Korea.

According to a report by the National Evidence-based Healthcare Collaborating Agency on dialysis modalities for ESRD, the total medical expenses in the Republic of Korea for HD was seven to nine times higher than that for peritoneal dialysis, and the annual cost per patient on HD was 4,100 to 7,400 USD higher than that per pa- tient on peritoneal dialysis [4]. This report indicates that vascular access-related expenses could contribute to the relatively high costs associated with $\mathrm{HD}$. According to the NHIC, the medical expenses for CKD was 1.56 trillion KRW in 2015 and 1.69 trillion KRW (equivalent to 1.51 billion USD) in 2016 [5]. The medical costs in the Republic of Korea for HD vascular access-related treatment were $2.3 \%$ of the total medical costs for CKD in 2015 and $2.5 \%$ of that cost in 2016. The United States Renal Data System statistics showed that yearly vascular access-related medical costs accounted for $1.2 \%$ of the total medical cost for CKD. In Japan, yearly vascular access-related medical costs were $1.4 \%$ of the total healthcare cost for CKD $[6,7]$. Our results indicated that the access-related medical expenses in the Republic of Korea accounted for a larger portion of the total CKD medical expenses compared with these other countries. The vascular access-related medical expenses in the present study re- 

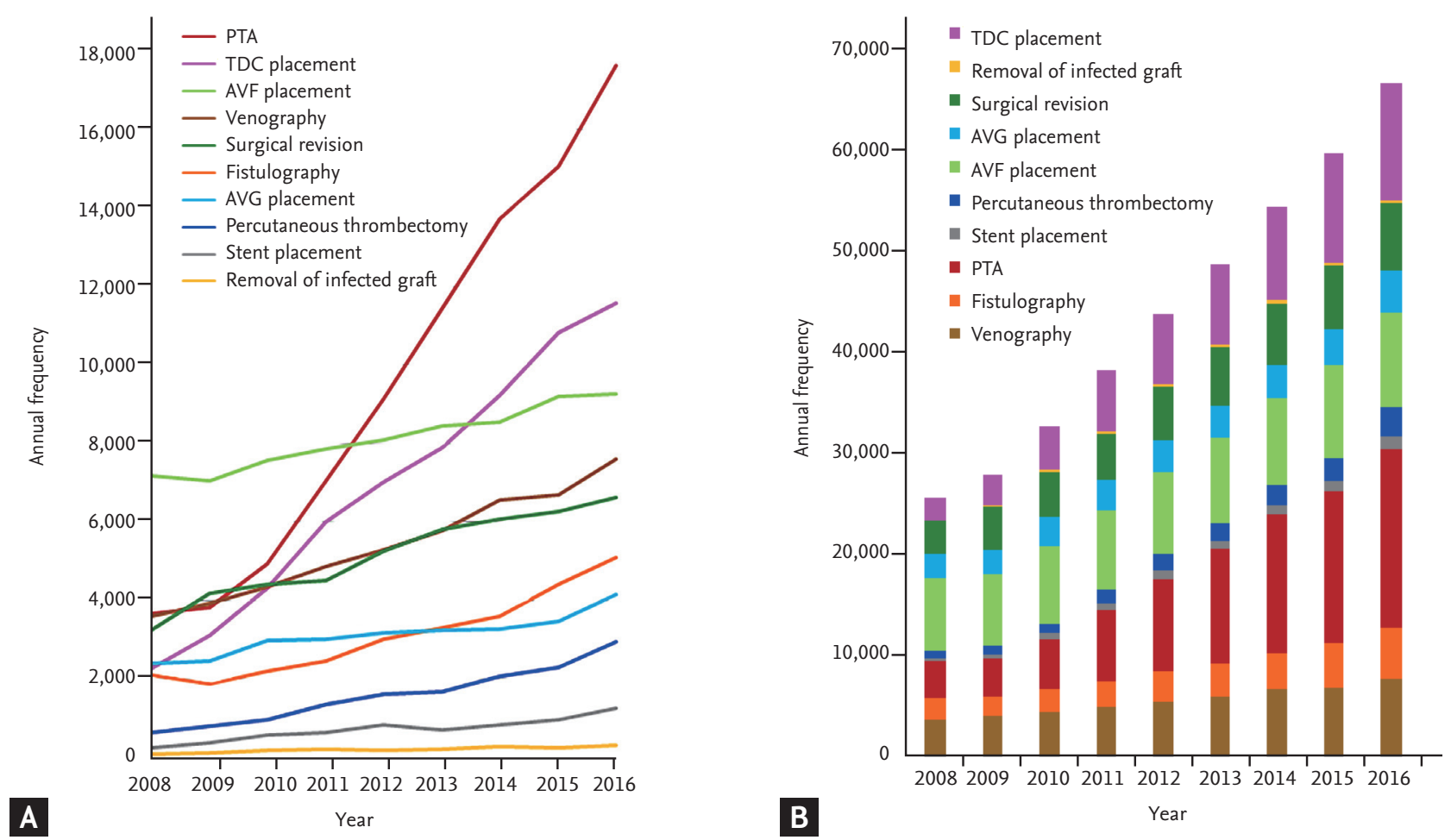

Figure 3. Annual frequency of procedures for vascular access-related treatments (2008 to 2016). (A) Trends in annual frequency of access-related procedures. The numbers of interventional or surgical procedures for vascular access increased with the growth of the number of patients on hemodialysis. (B) Trends in the proportion of annual frequency of access-related treatments. The increase in the number of percutaneous transluminal angioplasties was remarkable, leading the total growth in the number of procedures. PTA, percutaneous transluminal angioplasty; TDC, tunneled dialysis catheter; AVF, arteriovenous fistula; AVG, arteriovenous graft.

flected the procedure cost, and higher costs for vascular access are expected for inpatient services. Furthermore, the data did not include procedures and materials that were not covered by the insurance reimbursement, so the actual medical expenses for vascular access-related treatments are likely greater than the estimates in our study.

The increasing cost of access-related treatment is largely due to a steep increase in the medical costs associated with PTA. The annual frequency of PTAs has increased more rapidly than that of other access-related procedures. The increase in frequency of PTAs per patient has led the growth of the number of vascular access-related procedures. Patients with the highest frequencies of PTAs underwent more than 11 PTA procedures during their HD period, and the interval between PTAs shortened and converged on 90 days. The recent growth in the number of PTA cases could be due to an increase in the number of preemptive PTA procedures performed on a regular schedule as well as the increase in vascular access surveillance, which has been encouraged through the nationwide dialysis clinic assessment program. Some interventionalists routinely schedule repeat PTAs for patients on HD every 3 months. These regular, preemptive PTAs might explain the peak interval of 90 days. The National Kidney Foundation-Kidney Disease Outcomes Quality Initiative (NKF-KDOQI) guidelines emphasize that preemptive PTAs should be performed if an anatomical abnormality is associated with clinical or physiological abnormalities, such as decreased access blood flow, elevated venous pressure, decreased dialysis dose, or abnormal physical examinations, which should return to within acceptable limits following the intervention [8]. However, this consideration of clinical and physiological parameters is often overlooked in clinical practice. Several recent prospective randomized studies suggested that preemptive angioplasty failed to prevent access thrombosis $[9,10]$, 


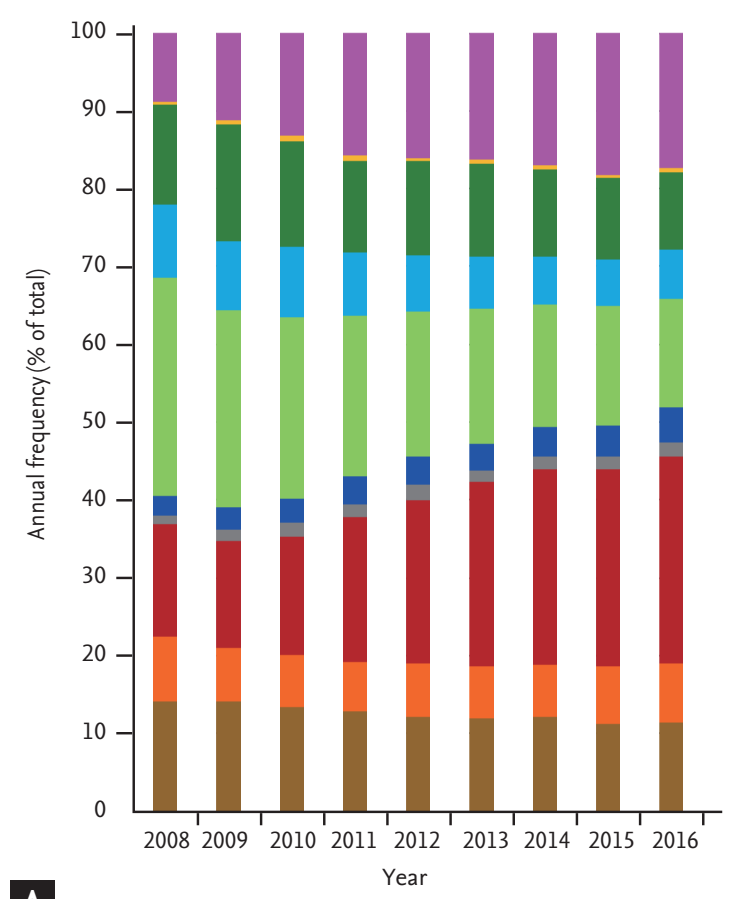

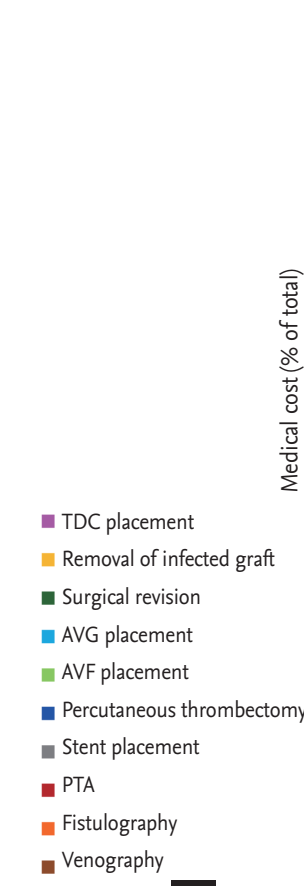

B

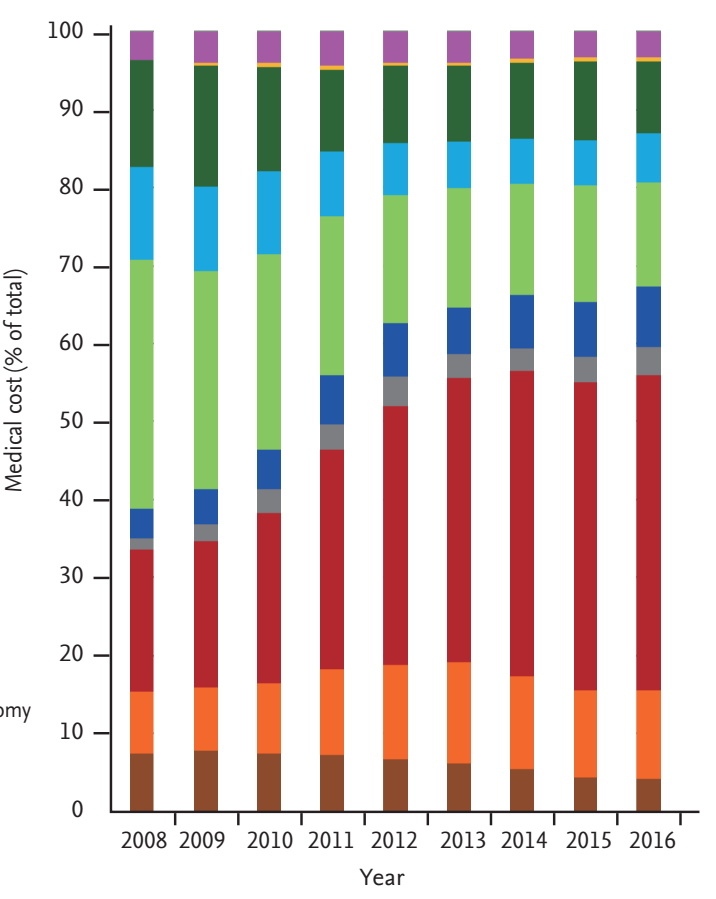

Figure 4. Trends in the percentage of frequency and medical costs of each procedure relative to total treatment procedures (2008 to 2016). (A) Trends in the percentage of frequency of each procedure relative to total treatment procedures. (B) Trends in the percentage of medical cost of each procedure relative to total treatment procedures. TDC, tunneled dialysis catheter; AVG, arteriovenous graft; AVF, arteriovenous fistula; PTA, percutaneous transluminal angioplasty.

and a systematic review by Ravani et al. [11] reported that preemptive angioplasty for stenosis in a functional AV access did not improve the longevity of the access. While preemptive angioplasty could be promising in AV fistulas, it may also raise the risk of procedure-related adverse events and costs [11]. There is currently insufficient evidence to support the practice of performing repeated preemptive angioplasty. Furthermore, the scheduling of regular PTAs every 3 months is not presently supported by any evidence-based data, so the necessity of treatment for stenosis should be decided based on the clinical and physiological aspects of the access.

Clinically, timely surgical correction provides much longer access patency in certain cases, such as the juxta-anastomotic stenosis and cephalic arch stenosis with frequent recurrence [12,13], and reduces unnecessary endovascular procedures, including angioplasty and stent deployment. The NKF-KDOQI guidelines recommended that patients should be re-evaluated for possible construction of a secondary fistula following each episode of access failure [14], and considered for surgi- cal revision prior to stent deployment unless they have a surgically inaccessible lesion or contraindications for surgery [8]. In the Republic of Korea, surgical thrombectomy, graft interposition, proximal re-anastomosis for juxta-anastomotic stenosis, aneurysmectomy, central transposition for cephalic arch stenosis, bypass procedure with a new graft, banding procedure for high flow fistula, and even proximalization of arterial inflow in distal hypoperfusion ischemic syndrome are uniformly classified as the same code of "repair of arteriovenous fistula (O2083)." The reimbursement benefit for repair of AV fistula is 428,010 to $467,670 \mathrm{KRW}$ (equivalent to 382.8 to 418.3 USD), which is only $58 \%$ of the reimbursement benefit for PTA. This inadequate reimbursement for surgical revision, including the disbursement of insurance benefits for surgical correction when PTA and surgical correction are performed together as a hybrid operation, could be a barrier to timely surgical revision and might cause healthcare providers to prefer endovascular treatment rather than trying surgical salvage of dysfunctional access, especially when surgical revision 

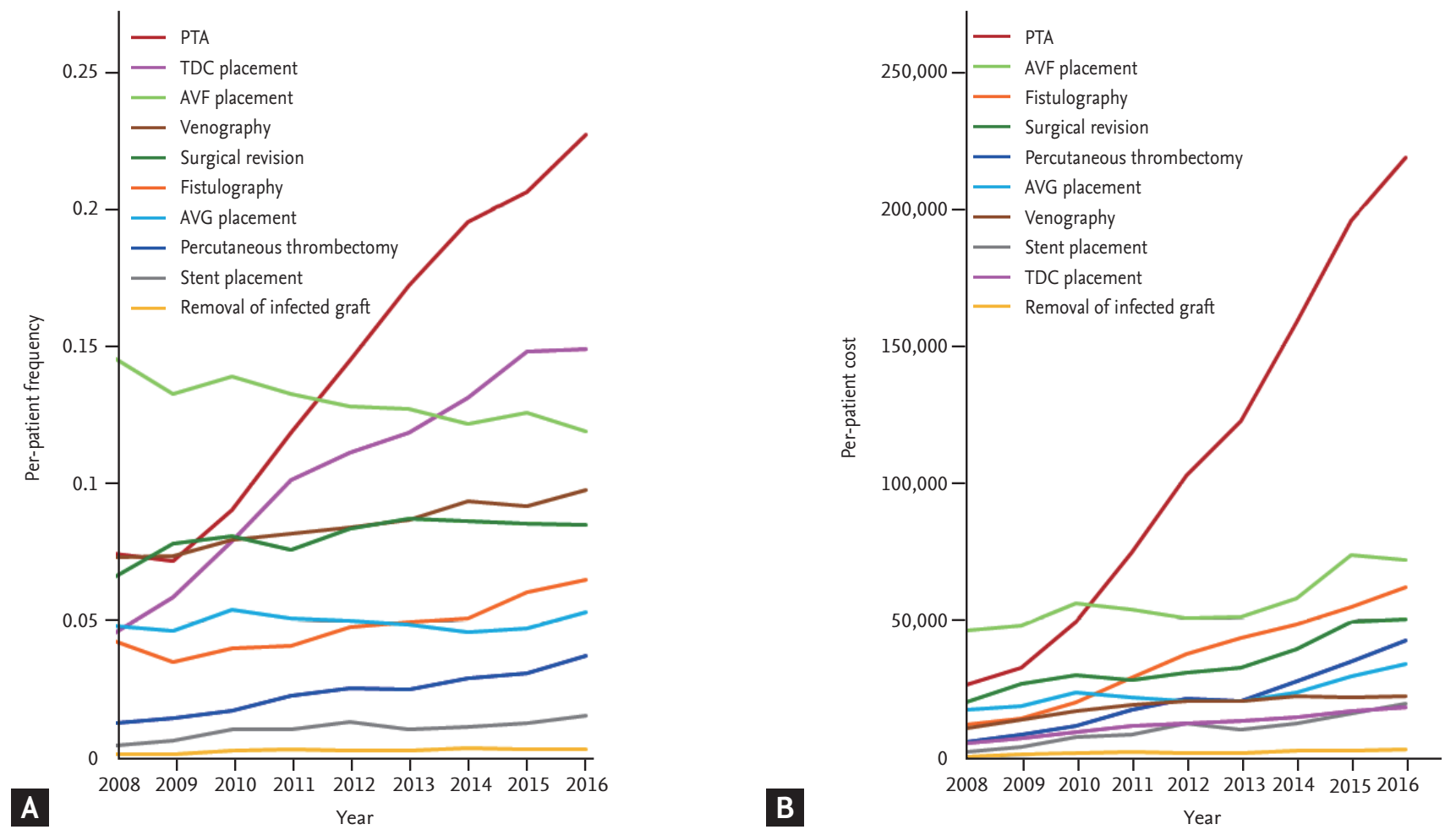

Figure 5. Trends in the per-patient frequency and medical cost of access-related treatment (2008 to 2016). The rapid growth in the number and cost of percutaneous transluminal angioplasties are attributed to the increase in the number of procedures per patient. (A) Trends in the per-patient frequency of access-related procedures. (B) Trends in the per-patient cost of access-related procedures. PTA, percutaneous transluminal angioplasty; TDC, tunneled dialysis catheter; AVF, arteriovenous fistula; AVG, arteriovenous graft.

is technically difficult. Rationalizing the reimbursement of surgical revision could therefore be an option to curb the rapid expansion of PTA expenses.

More than ever, endovascular treatment is preferred over surgical treatment, even when a surgical procedure is recommended. This trend is also reflected in the result of the present study, which showed the recent decline in the annual frequency of surgical revision in per-patient analysis. Regarding the current situation, the direct involvement of nephrologists in the monitoring of vascular access, timely detection of dysfunctional access, a policy for treatment modality, following of the functional aspects of access, and enhancement of interdepartmental cooperation could be important and productive goals for improving access care.

In the present study, the numbers of access placements included both the primary access creation in incident HD patients and secondary access creation in prevalent HD patients. The results therefore reflected the annual frequency of access creation itself but could not provide the information about the incidence of the primary access in incident HD patients or the prevalence of access types in prevalent HD patients. According to the Korean ESRD registry, the prevalence of arteriovenous fistula, arteriovenous graft, and TDC in the prevalent HD patients were $76 \%, 16 \%$, and $6 \%$ in the last years, respectively [15]. Because this study investigated ESRD patients on HD treatments, the result of this study could not include data on access creation in predialysis patients. In addition, this study was of a retrospective observational study, and the medical claims data could not provide information about non-benefit items that were not covered by insurance benefits. Thus, the medical expenditures in this study were an underestimation of the actual expenses. Because more non-benefit items are currently being incorporated into insurance benefit coverage in the Republic of Korea, our knowledge about the medical expenditures on diagnostic and therapeutic practices for HD vascular access will be more accurate in the future. 

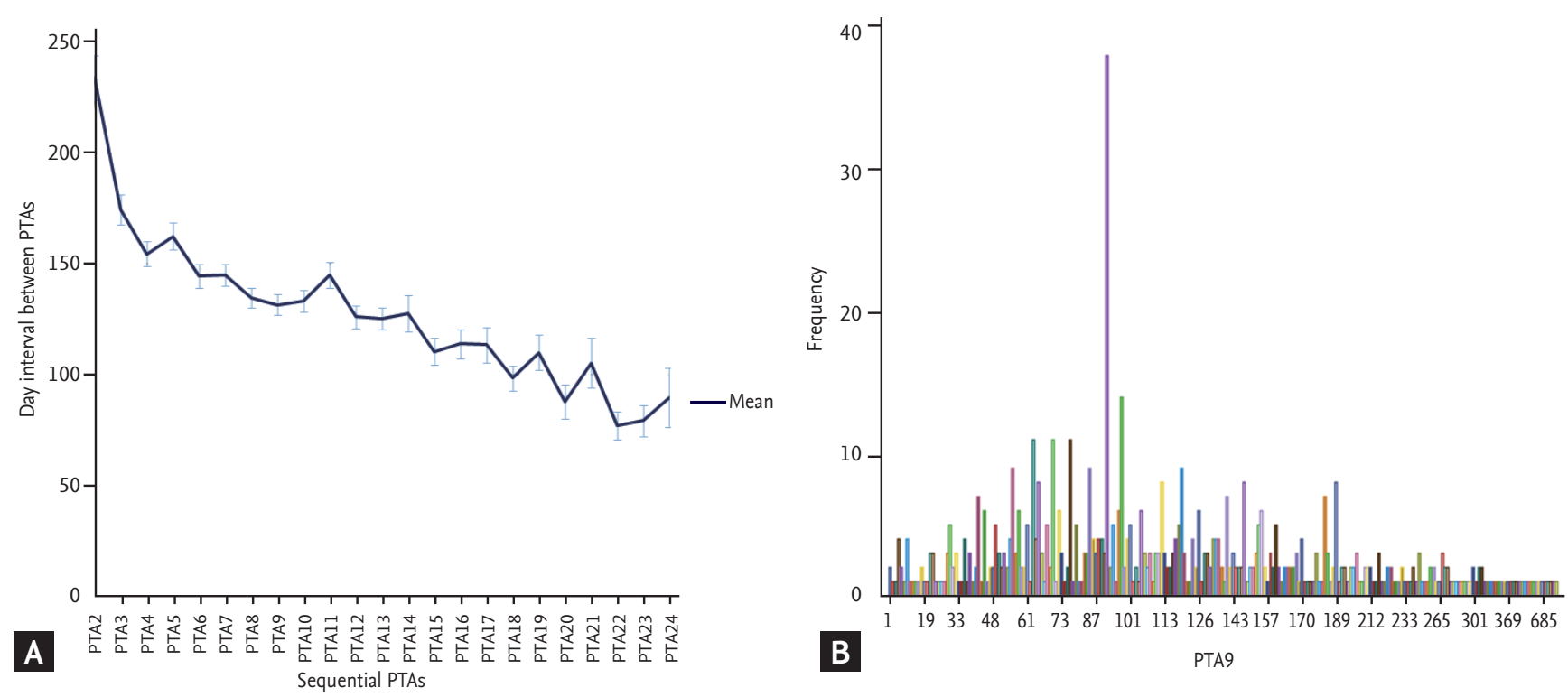

Figure 6. The time interval between successive percutaneous transluminal angioplasties (PTAs). (A) The time interval between successive PTAs among the patients who underwent more than 11 PTA procedures. The frequencies of time intervals converged on the 90-day interval as the procedure was repeated. The horizontal axis shows sequential PTAs; PTA2= the interval between the first PTA and the second PTA. The vertical axis shows the number of days in the intervals. (B) Frequencies of the time intervals between successive PTAs. This graph shows the frequency of the time intervals between the eighth and ninth percutaneous angioplasty. The most frequent time interval from the eighth PTA to the ninth PTA was 90 days.

\section{KEY MESSAGE}

1. The rapid growth in hemodialysis population has dramatically increased the number and medical expenses of access-related procedures.

2. Percutaneous transluminal angioplasty (PTA) was the main driver of the increase in access-related costs, becoming a financial challenge in the medical expenditures of chronic kidney disease management.

3. PTA should be performed when an anatomical abnormality is associated with functional abnormalities; therefore, active involvement of the dialysis staffs in the monitoring of clinical and physiological aspects of vascular access is important.

\section{Conflict of interest}

No potential conflict of interest relevant to this article was reported.

\section{Acknowledgments}

This research was supported by the Hallym University Research Fund 2014 (HURF-2014-58).

\section{REFERENCES}

1. Jin DC. Current status of dialysis therapy for ESRD patients in Korea. J Korean Med Assoc 2013;56:562-568.

2. National Health Insurance Service. National Health Insurance Program in Korea [Internet]. Wonju (KR): National Health Insurance Service, c2010 [cited 2018 Sep 6]. Available from: https://www.nhis.or.kr/static/html/wbd/g/ a/wbdgao401.html.

3. Statistics Korea. Korean Standard Statistical Classification of Disease [Internet]. Daejeon (KR): Statistics Korea, c1996 [cited 2018 Sep 6]. Available from: https://kssc.kostat. go.kr:8443/ksscNew_web/kssc/main/main.do?gubun=1\#.

4. National Evidence-based Healthcare Collaborating Agency. Outcomes research of peritoneal dialysis and hemodialysis for end-stage renal disease [Internet]. Seoul (KR): National Evidence-based Healthcare Collaborating Agency, c2017 [cited 2018 Sep 6]. Available from: https:// 
www.neca.re.kr/layı/program/S1T11C145/report/view. do?seq=223.

5. National Health Insurance Service. National Health Insurance Statistical Yearbook. 13th ed. Wonju (KR): National Health Insurance Service, 2017.

6. United States Renal Data System. 2017 USRDS Annual Data Report: Epidemiology of Kidney Disease in the United States. Bethesda (MD): National Institutes of Health, 2017.

7. Takemoto Y, Naganuma T. The economic issue of vascular access within the Japanese medical reimbursement system. J Vasc Access 2015;16 Suppl 10:S9-S12.

8. National Kidney Foundation. NKF-KDOQI clinical practice guidelines for vascular access. Am J Kidney Dis 2006;48(Suppl 1):S176-S276.

9. Dember LM, Holmberg EF, Kaufman JS. Randomized controlled trial of prophylactic repair of hemodialysis arteriovenous graft stenosis. Kidney Int 2004;66:390-398.

10. Moist LM, Churchill DN, House AA, et al. Regular monitoring of access flow compared with monitoring of venous pressure fails to improve graft survival. J Am Soc Nephrol 2003;14:2645-2653.
11. Ravani P, Quinn RR, Oliver MJ, et al. Preemptive correction of arteriovenous access stenosis: a systematic review and meta-analysis of randomized controlled trials. Am J Kidney Dis 2016;67:446-460.

12. Argyriou C, Schoretsanitis N, Georgakarakos EI, Georgiadis GS, Lazarides MK. Preemptive open surgical vs. endovascular repair for juxta-anastomotic stenoses of autogenous AV fistulae: a meta-analysis. J Vasc Access 2015;16:454-458.

13. Sigala F, Saben R, Kontis E, Kiefhaber LD, Forster R, Mickley V. Surgical treatment of cephalic arch stenosis by central transposition of the cephalic vein. J Vasc Access 2014;15:272-277.

14. National Kidney Foundation. NKF-KDOQI clinical practice guidelines for vascular access. Guideline 29: goals of access placement maximizing primary AV fistulae. Am J Kidney Dis 2001;37(Suppl 1):S169.

15. The Korean Society of Nephrology. Korean ESRD Registry 2018 [Internet]. Seoul (KR): Korean Society of Nephrology, c2016 [cited 2018 Sep 6]. Available from: http://www. ksn.or.kr/rang_board/list.html?code=sinchart_eng. 


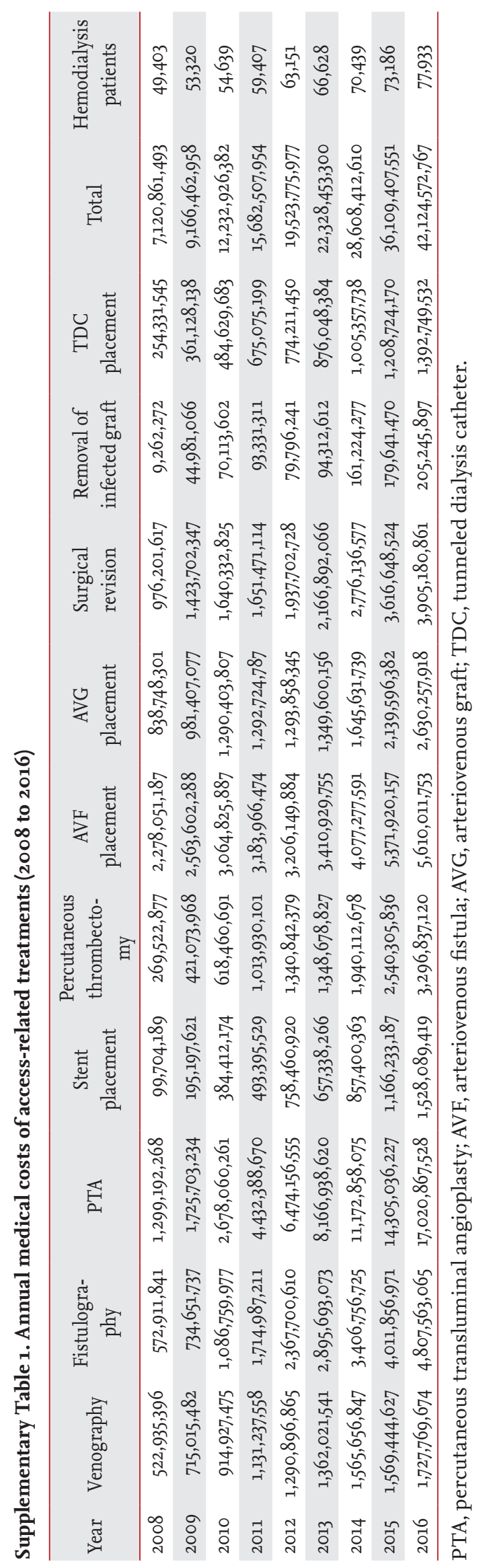




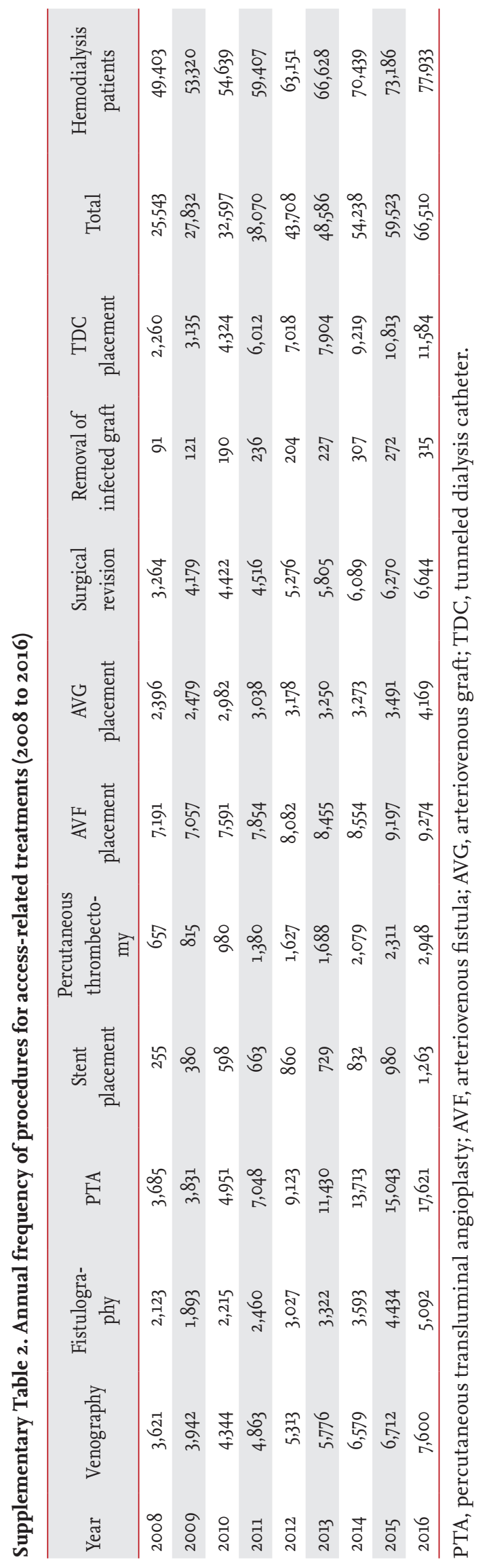




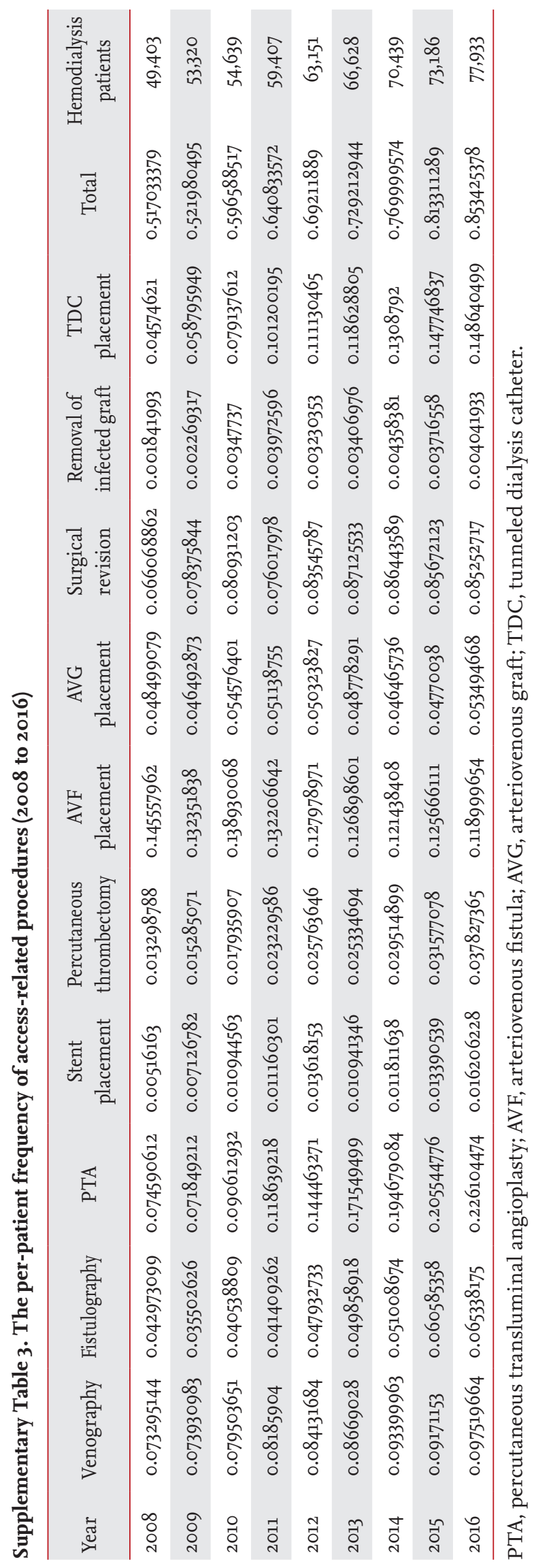




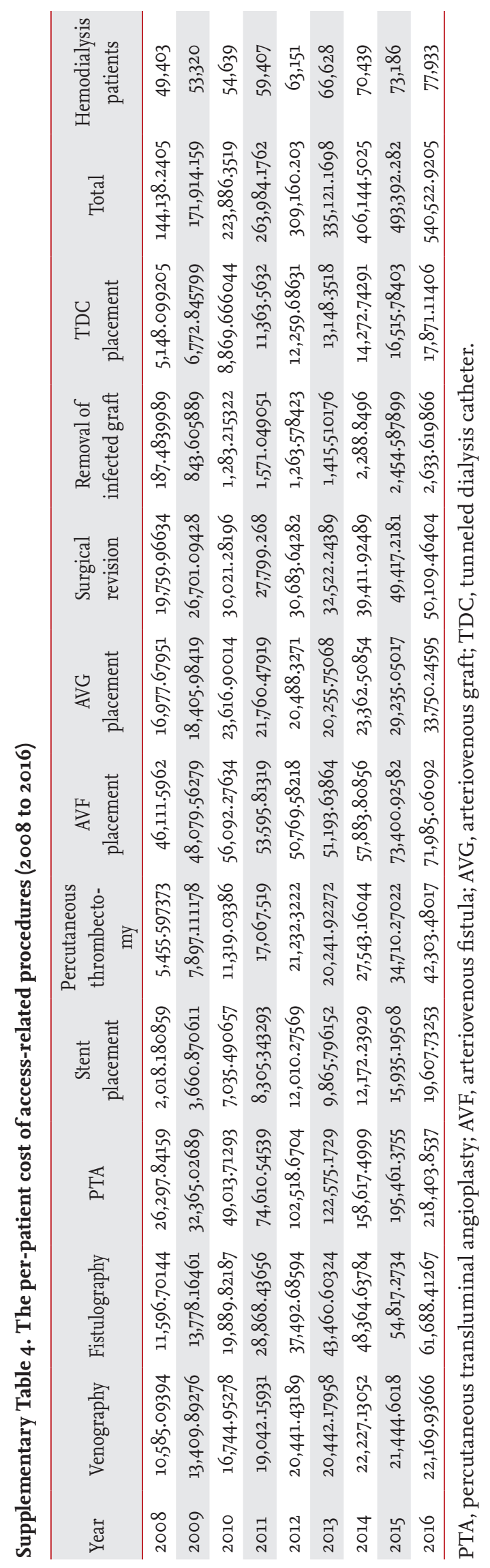




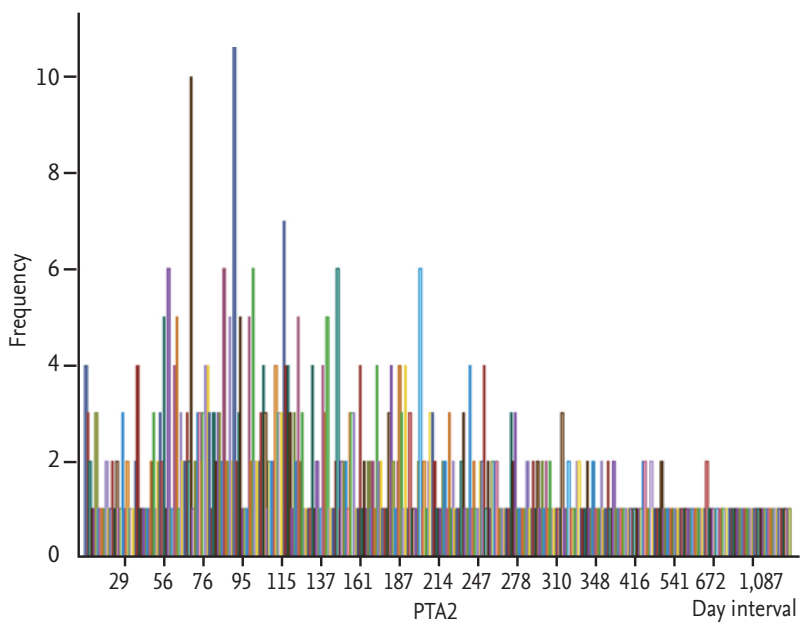

Supplementary Figure 1. Frequencies of the time intervals between the first and second percutaneous angioplasties. PTA, percutaneous transluminal angioplasty. 


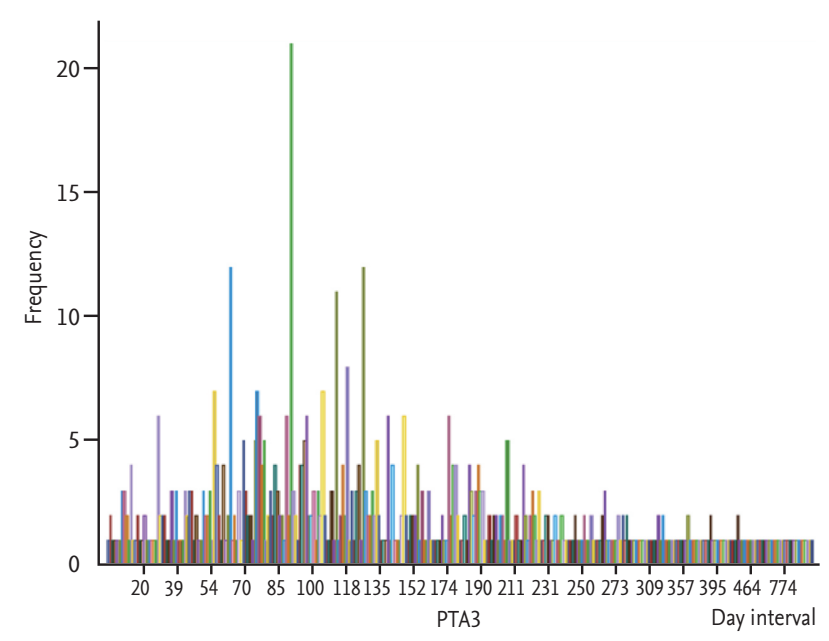

Supplementary Figure 2. Frequencies of the time intervals between the second and third percutaneous angioplasties. PTA, percutaneous transluminal angioplasty. 


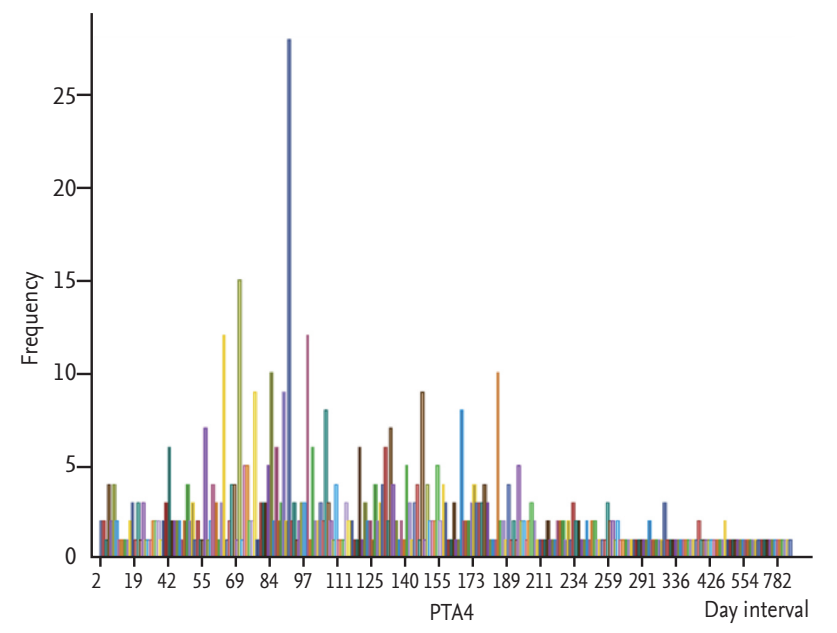

Supplementary Figure 3. Frequencies of the time intervals between the third and fourth percutaneous angioplasties. PTA, percutaneous transluminal angioplasty. 


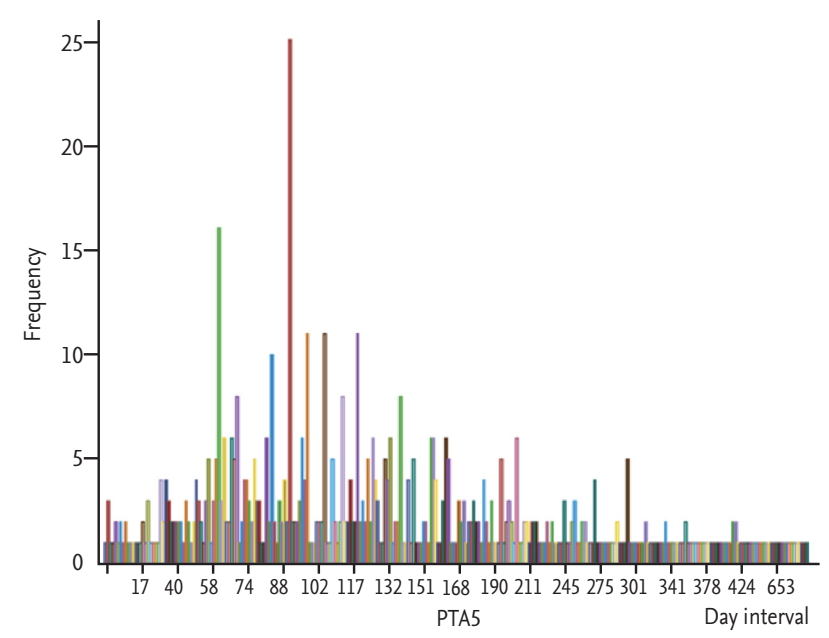

Supplementary Figure 4. Frequencies of the time intervals between the fourth and fifth percutaneous angioplasties. PTA, percutaneous transluminal angioplasty. 


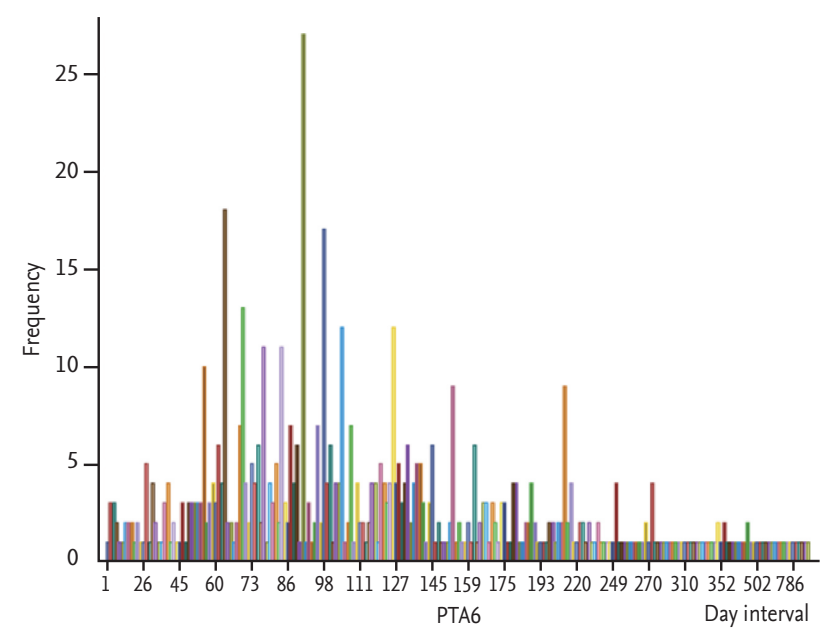

Supplementary Figure 5. Frequencies of the time intervals between the fifth and sixth percutaneous angioplasties. PTA, percutaneous transluminal angioplasty. 


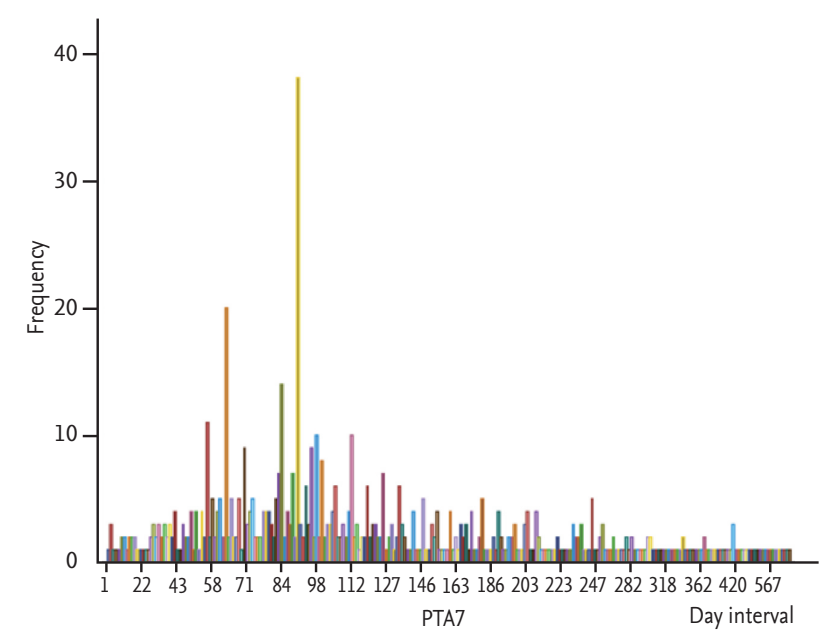

Supplementary Figure 6. Frequencies of the time intervals between the sixth and seventh percutaneous angioplasties. PTA, percutaneous transluminal angioplasty. 


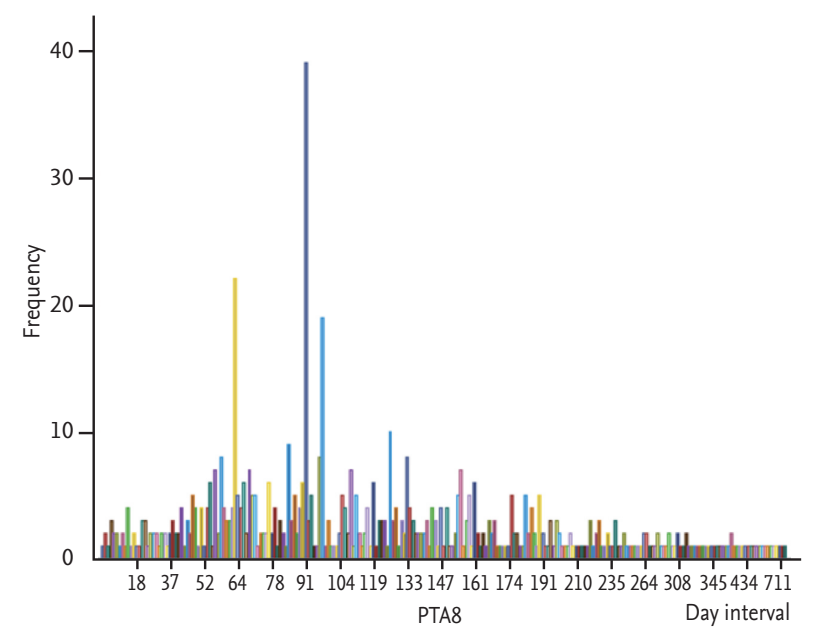

Supplementary Figure 7. Frequencies of the time intervals between the seventh and eighth percutaneous angioplasties. PTA, percutaneous transluminal angioplasty. 


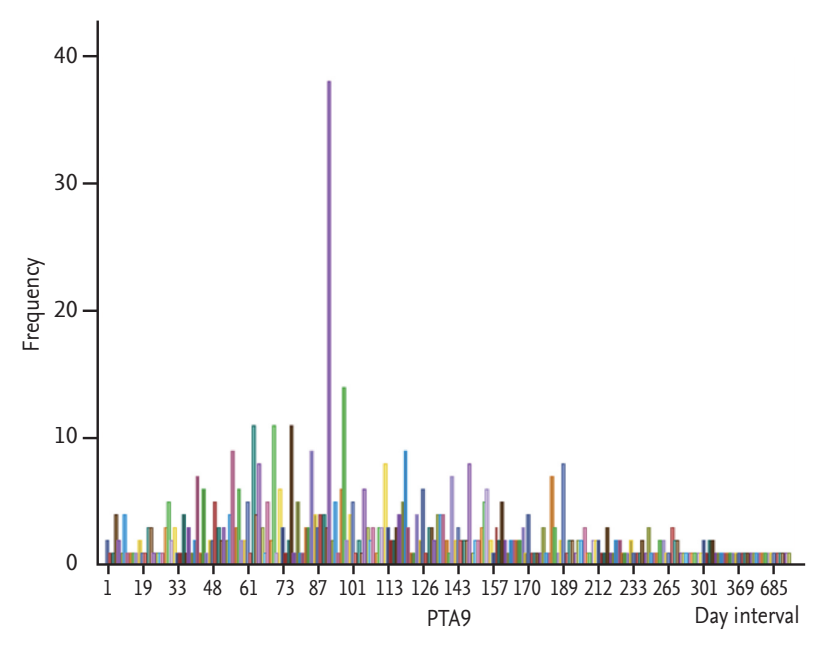

Supplementary Figure 8. Frequencies of the time intervals between the eighth and ninth percutaneous angioplasties. PTA, percutaneous transluminal angioplasty. 